\title{
Anomalous Behavior of Highly Active Helical Swimmers
}

\author{
Gouri Patil ${ }^{1 *}$ and Ambarish Ghosh ${ }^{1,2 *}$ \\ ${ }^{1}$ Department of Physics, Indian Institute of Science, Bangalore, India, ${ }^{2}$ Centre for Nano Science and Engineering, Indian Institute \\ of Science, Bangalore, India
}

Artificially designed self-propelled objects can allow studying active matter phenomena with great detail that is not possible in natural, e.g. biological systems. Here, we show experimental results on helical shaped, magnetically actuated, reciprocal swimmers, where the degree of randomness in the reciprocal sequence plays an important role in determining their effective motility. Here, for the first time we show the results at high activity levels where the degree of randomness is further affected by the presence of the surface, which in turn results in a non-monotonic increase of motility as a function of magnetic drive. It will be interesting to extend these studies to denser systems where the swimmers can interact with each other through hydrodynamic forces.

OPEN ACCESS

Edited by:

Ayan Banerjee,

Indian Institute of Science Education and Research Kolkata, India

Reviewed by: Marco G. Mazza,

Loughborough University, United Kingdom

Christian Maes,

KU Leuven, Belgium

*Correspondence:

Ambarish Ghosh ambarish@iisc.ac.in

Gouri Patil

gouriiisc.ac.in

Specialty section: This article was submitted to Interdisciplinary Physics, a section of the journal

Frontiers in Physics

Received: 11 November 2020

Accepted: 18 December 2020

Published: 20 January 2021

Citation:

Patil G and Ghosh A (2021) Anomalous Behavior of Highly Active Helical Swimmers.

Front. Phys. 8:628276.

doi: 10.3389/fphy.2020.628276
Keywords: magnetic helical swimmers, reciprocal swimmers, active matter, diffusivity enhancement, artificial swimmers

\section{INTRODUCTION}

Much of the microscopic world is dominated by living organisms whose sustenance is strongly dependent on their ability to move in fluidic environments. The importance of motility is observed in bacteria's search for food or spermatozoa's struggle to reach the ovum. However, the small size of the microorganisms results in a large surface ( $\propto$ frictionalforce) to volume ( $\propto$ inertialforce) ratio $\left(\sim 10^{4}\right)$. At such low Reynolds numbers, the role of inertia is entirely negligible, which has resulted in the evolution of novel swimming strategies adopted by the microorganisms to overcome the fluidic drag [1]. A particular strategy that cannot lead to motility at microscopic scales is reciprocal motion. As Purcell [2] describes Scallop theorem in his "Life at low Reynolds number"; “. . .I change my body into a certain shape and then I go back to the original shape by going through the sequence in reverse. At low Reynolds number, everything reverses just fine ... So, if the animal tries to swim by a reciprocal motion, it can't go anywhere". This kind of motion in bulk Newtonian medium cannot result in net displacement of an individual swimmer.

Indeed, one way to work around [3] the Scallop theorem is to adopt non-reciprocal swimming strategies. For instance, many bacteria swim by rotating chiral (helical) flagella which leads to translation [4]. Similar strategies have been used to make synthetic swimmers at small scales, where the rigid magnetic helices propel in the desired direction due to the rotation induced by a rotating magnetic field $[5,6]$. Flexible filaments subjected to whip-like beating pattern can result in net motion as well $[7,8]$. Apart from these strategies to break the back and forth symmetry, the physical environment can also result in the violation of the Scallop theorem. For instance, two bodies exhibiting reciprocal actuation with a phase difference exploit the unsteady hydrodynamic flows [9] created by each other to achieve collective locomotion. Alternately, non-Newtonian properties, e.g., shear thinning [10] or viscoelastic nature of the surrounding medium can aid in the locomotion of reciprocal swimmers. The fluid elastic stresses [11] or difference in forward and backward strokes of reciprocal swimming $[12,13]$, can be the source of motion in a non-linear viscoelastic environment. 

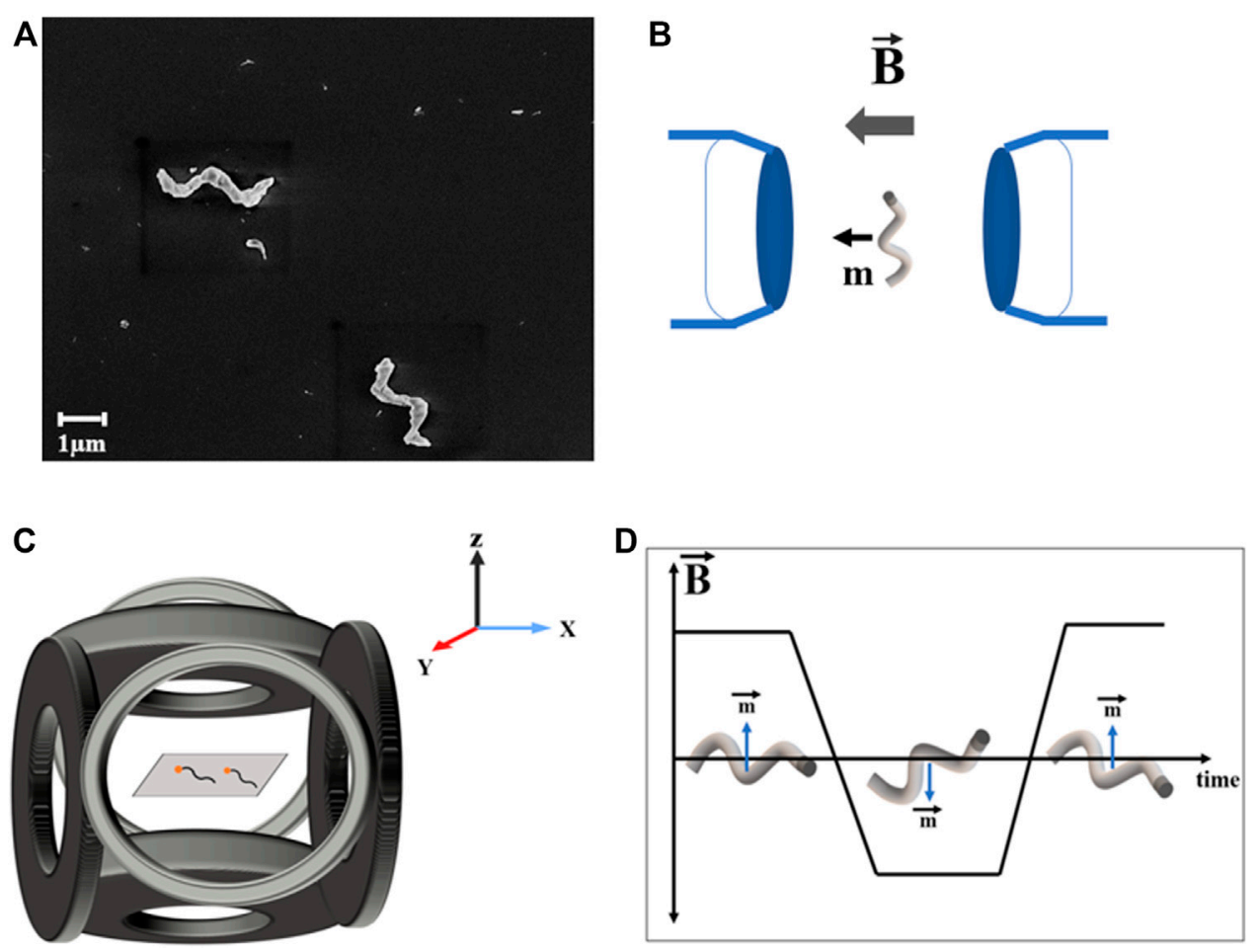

FIGURE 1 | (A). SEM image of the helical swimmers. (B). A dc-field magnetizes the swimmer with permanent magnetic moment along the short axis. (C). The schematic of a triaxial Helmholtz coil, which enables a uniform magnetic field at the center of the coil. The swimmers are placed in the sample chamber at the center of the coil. (D). The oscillating magnetic field in the $z$-direction causes the back-forth motion of the propeller.

In this article, we examine a system of artificial reciprocal swimmers built on the experimental platform of rigid magnetic helical nano-propellers, which move under the influence of a rotating magnetic field [5]. As shown extensively before, this is a versatile, multifunctional bio-manipulation [14-16], delivery [17, 18] and sensing system [19-21]. Typically, this is a driven system where the sense (clockwise, CW or counterclockwise, CCW) and the plane of rotation of the magnetic field determines the direction of propulsion depending on the chirality of the helix [22]. This is unlike the other self-propelled synthetic swimmers, where the direction of non-interacting motile objects are independent of each other [23, 24]. The helical propulsion system can be changed from driven to active [25] system by simply changing the magnetic drive from rotating to oscillating field configuration, where the plane of oscillation is perpendicular to the plane (within the microfluidic chamber) where the propellers are experimentally maneuvered. As a result, the orientation of the propellers are independent of the external field, and they undergo back and forth motion, brought about by the CW-CCW rotation of the helices. As shown previously [26, 27], a subtle counterplay between the magnetic drive and weight asymmetry of the helices governs the rotational dynamics of the propellers. Note that the time average displacement of a reciprocal helical swimmer is zero in the absence of any diffusion. However, in the presence of thermal fluctuations in the surrounding fluid, these objects can show considerable diffusion compared to their passive counterparts [26, 28], which is caused by the orientational fluctuation of the swimmer during the back and forth motion. In summary, the experimental system of reciprocal propellers is a zero torque, zero force active system and the activity can be regulated via frequency of the external magnetic field. The activity of this system can be tuned easily by changing the magnetic drive parameters, and the experimental studies can be performed over long duration which make them an attractive potential system to study the emergence of various interesting active matter properties $[29,30]$ such as the roles of confinement [31, 32], surface effects [33, 34], and collective interaction [35].

In this study, we will be specifically discussing the enhancement in diffusivity of the reciprocal swimmers as a function of frequency and the parameter- "degree of randomness", described later. In our previous studies [25, 26], the enhancement of diffusivity at lower frequencies of actuation was investigated, where the diffusivity increased by $78 \%$ compared to the passively diffusing structures. As we show here, new dynamical behavior of the swimmers emerge at higher activity levels (increase in diffusivity up to 300\%), including the role of the surface of the fluidic chamber, that was neglected before.

\section{RESULTS AND DISCUSSION}

The system of swimmers used here consists of helical structures made of silica and a magnetic material that are fabricated via Glancing Angle Deposition (GLAD) method [36]. These helices 

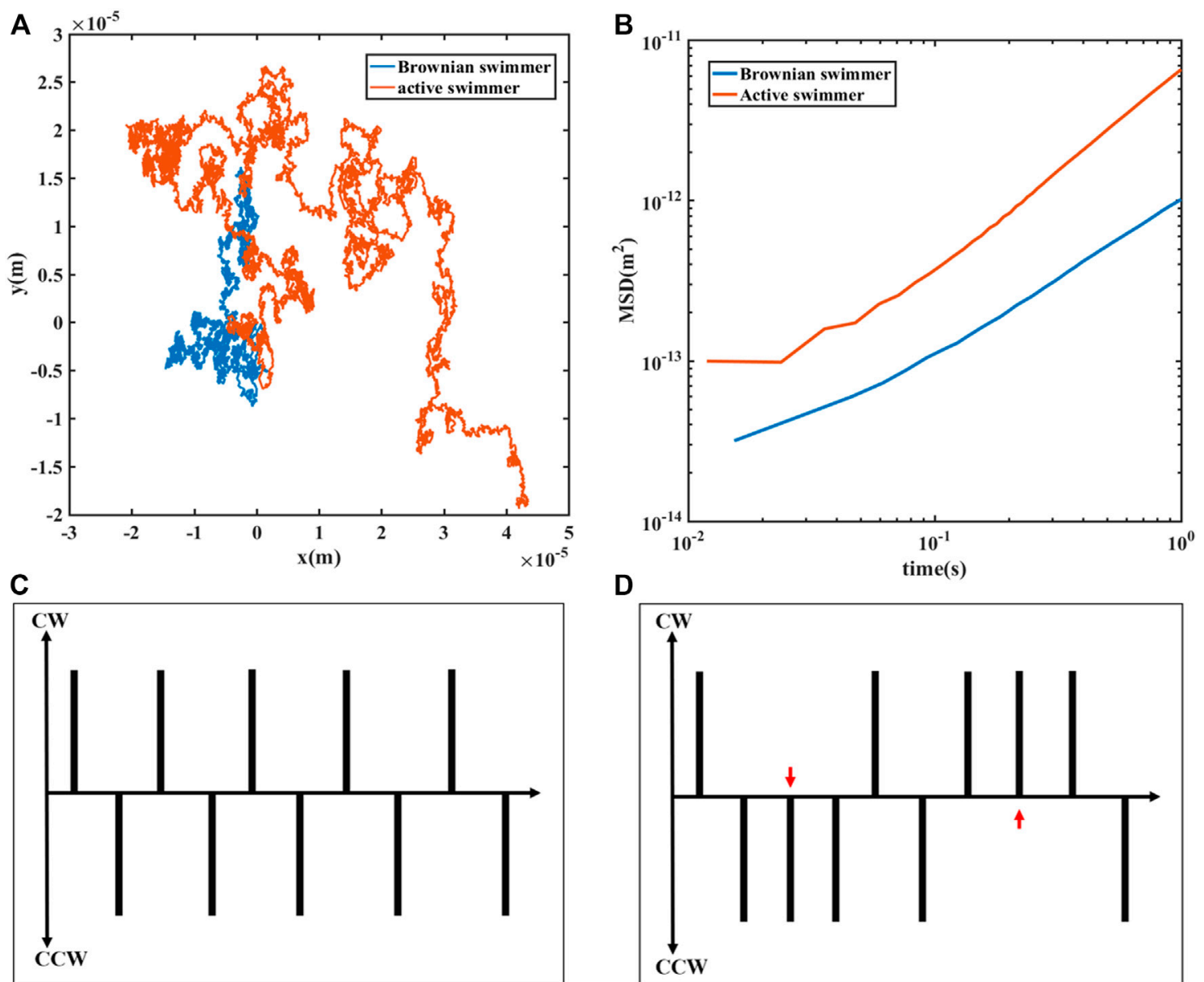

D

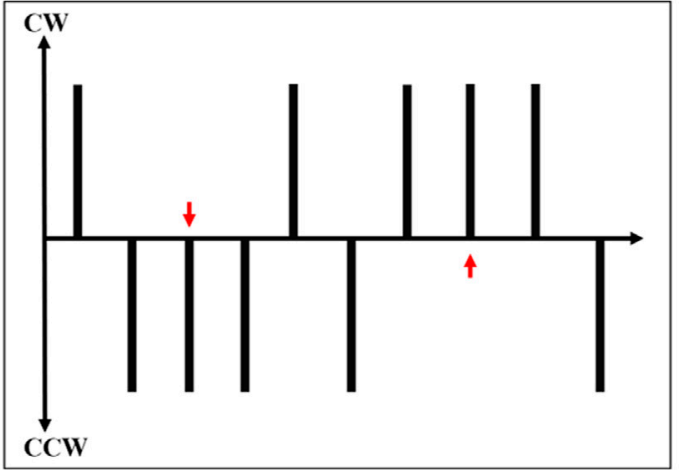

FIGURE 2 | (A). The typical trajectory is comparing the Brownian and an active propeller. The propeller (red curve) is performing reciprocal motion as a response to an external field of amplitude $80 \mathrm{G}$ and frequency $10 \mathrm{~Hz}$. (B). Comparison of enhancement in diffusivity for the Brownian and active propeller. (C). The enhancement of diffusivity is closely related to the ' $q$ ' parameter, which is a ratio of broken reciprocity. The table shows a perfect sequence of clockwise-counterclockwize rotations about the long axis of the propeller. (D). The schematic indicates two instances (red arrows) where the sequence is broken, giving a q value of 2/10.

are grown on a substrate patterned with an array of pillars having a diameter of $200 \mathrm{~nm}$. The helical body is made of silica with magnetic material Iron and Cobalt incorporated at one end [37] of the structure. These 3-micron length propellers are later magnetized along their short axis to have a permanent magnetic moment [38], as shown in Figure 1B. The swimmers are sonicated in deionized water and laid down on a substrate to image them via SEM, as shown in Figure 1A. The swimmers are suspended in deionized water in a microfluidic device of thickness $20-25 \mu \mathrm{m}$, and within few minutes, the swimmers settle down close to the bottom surface, approximately $1 \sim 2$ microns above the bottom glass surface. For magnetic actuation, a triaxial Helmholtz coil is used where the three pair of coils in xyz direction are powered by amplifiers, thus producing a spatially uniform magnetic field at the center of the coil. The microfluidic chamber is placed at the center of triaxial coil coinciding with the imaging plane of the microscope. A rotating magnetic field due to the $\mathrm{X}, \mathrm{Y}, \mathrm{Z}$ coils drives the swimmers and can be controlled effectively via the strength and frequency of the field. By driving the helices along a specific direction in the xy plane, we find a linear relationship between the translational speeds and the frequencies of the rotating field from where the hydrodynamic pitch of the propellers is estimated to be $p_{h}=600 \mathrm{~nm}$. Note the linear relationship is only valid at low frequencies where the drag is not larger than the maximum available magnetic torque. For reciprocal actuation, a sinusoidal signal is fed only to the pair of z-coils resulting in an oscillating field in the $z$-direction. It is to be noted that the sample is placed in the xy imaging plane, whereas the external magnetic field is perpendicular to this imaging plane. Hence, the permanent magnetic moment vector along the short axis of the swimmer follows the external oscillating magnetic field and turns the propeller clockwise (CW) and counterclockwise (CCW) alternately. This would also result in the propeller moving forward and backwards by a distance of $p_{h} / 2$ in body frame. Note, the swimmer is subjected to thermal fluctuations in the xy plane, and hence the directionality is not governed by any external force.

Previously, in a theoretical work, Lauga [28] discusses the case of reciprocal swimmers. He found that for time scales larger than 

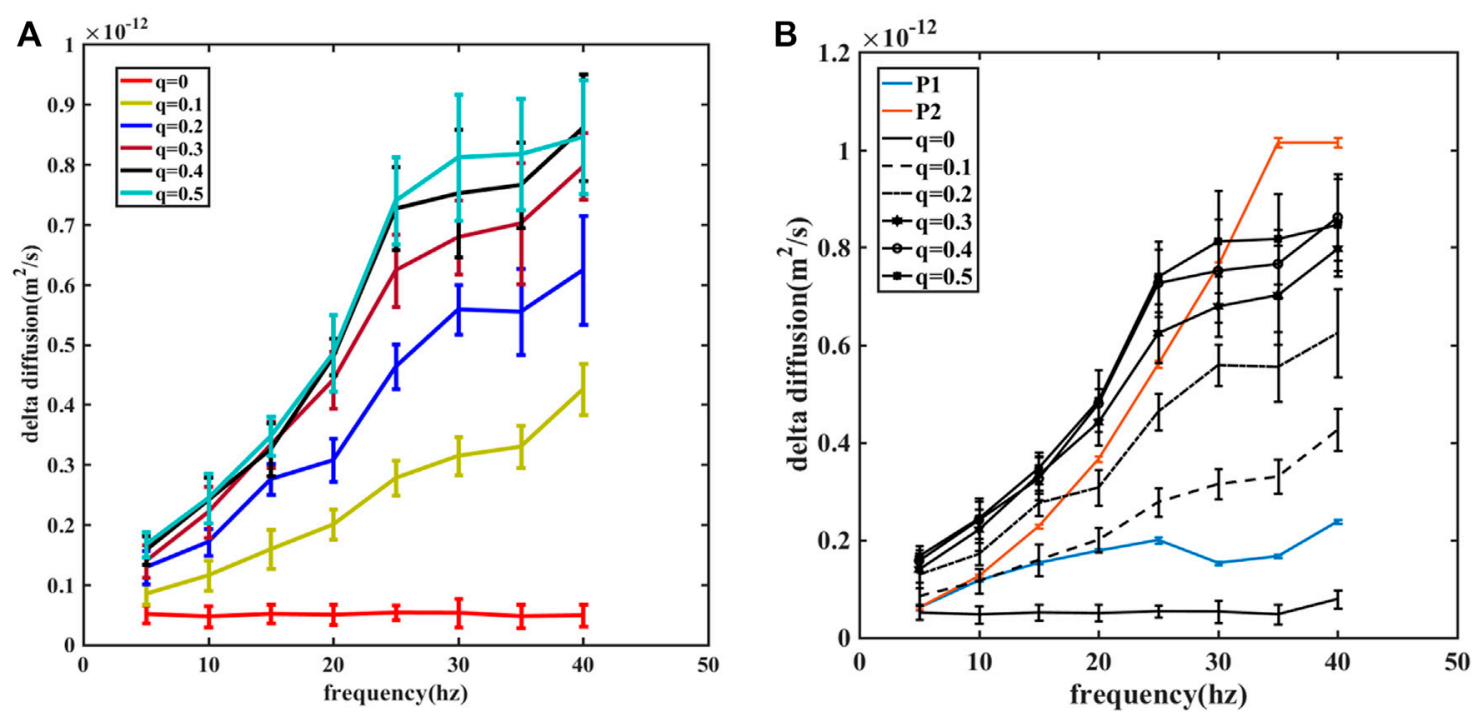

FIGURE 3 | (A). Simulations are carried out to delineate the effect of ' $q$ ' on the enhancement of diffusivity. As the frequency of the field is increased, the enhancement increases too. However, the introduction of q allows for much higher activity even at the same frequency. (B). Enhancement of two sample swimmers is plotted onto the simulated results. It is evident that the P1 (red) and the P2 (blue) swimmers behave differently.

the rotational diffusion, swimmers show significant enhancement in diffusivity compared to Brownian motion. The swimmer under such reciprocal actuation experiences enhanced fluctuations with effective diffusion constant $D_{r e c}$ which is always greater than the Brownian diffusion constant $D_{k_{B} T}$. This effect is dependent on the time period of reciprocal actuation, and the orientational diffusion time. In case of Mandal et al [26], the predicted enhancement in diffusivity is experimentally realized with a system of helical swimmers. They discuss the detailed dynamics of the swimming where the role of asymmetric weight distribution along the propellers' body is explained. Their numerical simulation took into account that the swimmers are subjected to a sinusoidal drive and the effect of a parameter $q$, namely degree of randomness; discussed in the following section. However, both these approaches rely on modeling of the swimmer in bulk. While for the experiments reported in this article, we have observed anomalous rise of D for the same swimmer at higher frequencies for which the present theories do not provide an explanation. We believe this needs to be addressed by incorporating an effect of surface, which has not been accounted for in any of the theories.

The effect of enhancement is shown in the two trajectories in Figure 2A corresponding to a propeller under external actuation and another undergoing Brownian motion, tracked using a 50x magnification objective lens and a camera. Both the trajectories are plotted for $120 \mathrm{~s}$, and one of the swimmers was subjected to 80 Gauss of magnetic field strength oscillating at $10 \mathrm{~Hz}$. The corresponding Figure 2B shows the mean squared displacement curves for both the propellers, where one (red) shows an increased diffusivity compared to Brownian propeller (blue). The $D_{\text {rec }}$ being $2.76 \times 10^{-13} \mathrm{~m}^{2} \mathrm{~s}^{-1}$ and $D_{k_{B} T}$ being $1.5 \times 10^{-13} \mathrm{~m}^{2} \mathrm{~s}^{-1}$, the $\Delta D$ is around $1.26 \times 10^{-13} \mathrm{~m}^{2} \mathrm{~s}^{-1}$. Ideally, the swimmers should follow a sequence of CW and CCW turns, resulting in a perfect reciprocal sequence (e.g., see Figure 2C). However, experimentally it has been observed that swimmers do not show a perfect reciprocal sequence. For instance, as shown in Figure 2D, the sequence of CW-CCW has been interrupted twice (red arrows) and this is due to the rotational diffusion around the long axis of the propeller which introduces a degree of randomness [26], " $q$ ". In this case, the $q$ takes the value of $2 /$ 10. The behavior of the propellers at lower frequencies and the importance of $q$ was discussed in the prior work [26]. Here we extend this to much higher frequencies and point out a new, distinct effect where the value of $q$ is found to depend on the actuation frequency for certain swimmers. Note the degree of randomness $q$ has a direct effect on the enhanced diffusivity of the swimmers.

As shown in Figure 3A, we performed numerical simulations to investigate the relation between the frequency of the magnetic field, degree of randomness and $\Delta D$. The $\Delta D$ always increases with frequency (activity), which is larger for higher values of $q$. Physically, this dependence on $q$ can be understood as a larger step size of the back and forth motion and therefore, more significant enhancement of the diffusivity. We also show experimental results of $\Delta D$ as a function of frequency, and this is shown in Figure $\mathbf{3 B}$ for two propellers, P1 and P2. The critical point to note in Figure $\mathbf{3 B}$ is the difference between the P1 (blue) and P2 (red) propellers. While the $\Delta D$ for P1 increases monotonically with frequency, it is clear that the values of $q$ for this propeller lies between 0 and 0.1 , without any apparent dependence of $q$ on the applied frequency. The swimmer named P2 appears to have a changing $q$ with frequency.

Before delving into the discussion regarding this dependence of $q$ on frequency, we will discuss a way to determine this degree of randomness experimentally. Indeed, one way is to image and 


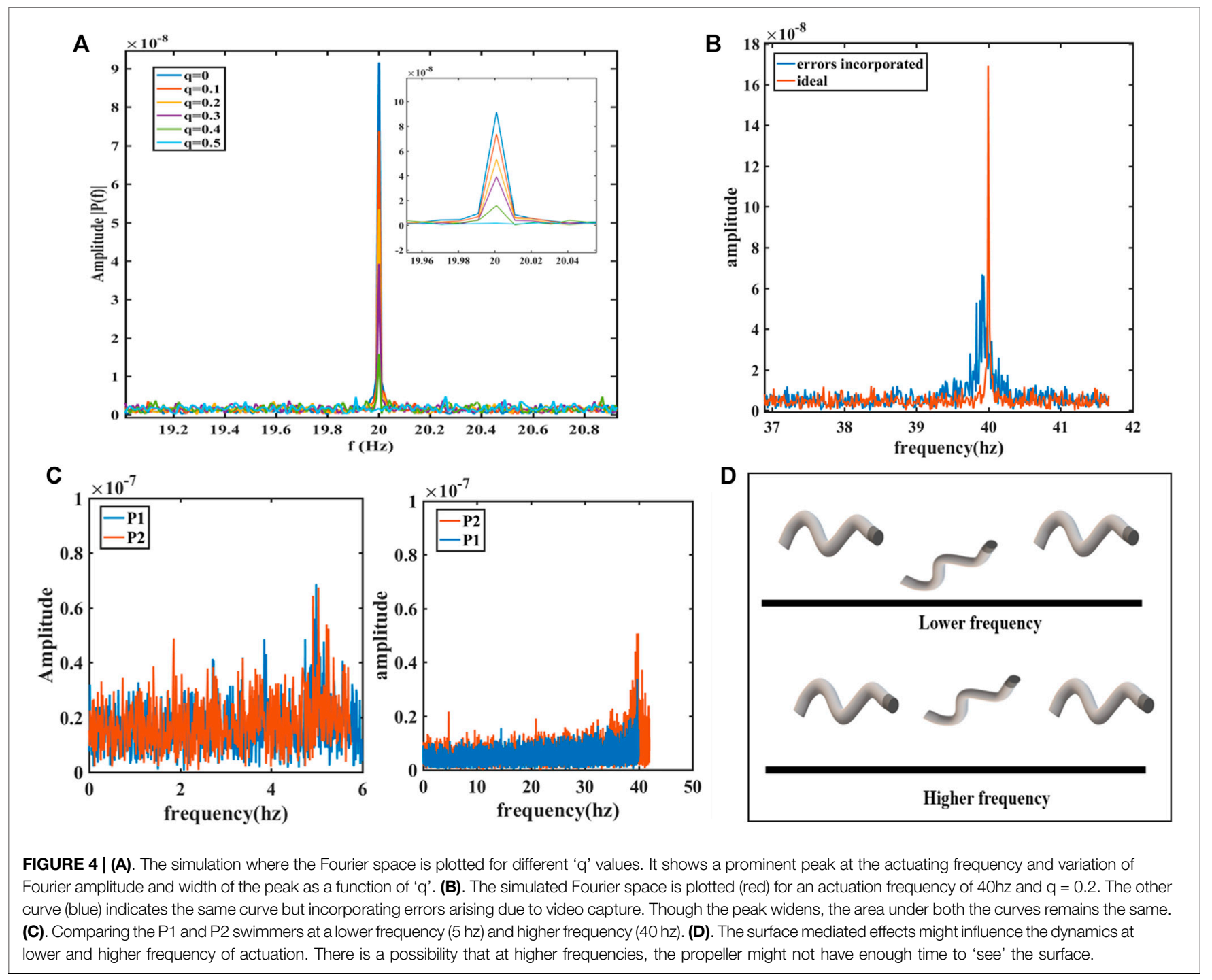

analyze the rotational dynamics of the swimmer, but this may not be possible to perform for experiments where turning of the swimmer may be difficult to observe, e.g. when the helix dimensions are smaller. Here, we show that the $q$ value can be directly deduced from the time domain Fourier space of the body frame displacements along the long axis of the swimmers. The positions of a swimmer in the lab frame can be tracked from the video. The algorithm detected the propeller as a rod, and its orientation with respect to the $x$-axis of the lab frame was recorded. It is necessary to record the video at a sampling rate equal to or more than $2 f$, $\mathrm{f}$ being the frequency of actuation. The displacement value along long axis of the propeller typically represents the step size in half a cycle of actuation. This provides us with a series of alternately positive and negative displacements corresponding to the back-forth motion. Typically, a Fourier transform of such a series must result in a peak at the actuating frequency, where the amplitude of the peak is inversely related to the $q$. This is expected because, with an increase in $q$, the periodicity is disrupted and results in a decrease in the peak amplitude. The fast Fourier Transform was implemented in MATLAB for a sequence of $\Delta x$ displacements of total length $\mathrm{T}$.

$$
y(k)=\sum_{t=1}^{T} \Delta x(t) e^{(-2 \pi i)(t-1)(k-1) / T},
$$

In Figure 4A, the simulation shows a peak at the actuating frequency, here $20 \mathrm{~Hz}$ for total time $\mathrm{T}=100 \mathrm{~s}$ and the variation of peak amplitude with $q$. However, Figure 4A represents an ideal case where the sampling rate of data (here the images per second) has been assumed to be constant.

On the other hand, during the recording of the experiments, the camera might introduce errors, such as a possibility of dropping of frames or variation in the frame rate. We simulate a condition where if $F_{s}$ is the rate per second at which images are captured, $\delta$ is the error introduced as the 
fluctuation in the frame rate. A $\pm 10 \%$ probability of frame rate being $F_{s} \pm 2$ is considered using randomly drawn numbers from uniformly distributed set between $[0,1]$. The results as shown in Figure 4B, the Fourier peak grew wider and shorter than the ideal case. However, the area under the curve remained same in both the cases; highlighting a vital observation that despite the shortcomings in imaging, Fourier space of the displacements is an excellent way to estimate $q$.

We believe the anomalous behavior of propeller P2 which exhibits a variation in $q$ with frequency (as shown in Figure 3B) can be explained by taking into account the role of surface of the microfluidic chamber. As observed in previous study [26], two main factors affect the dynamics of the helical swimmers. First is the asymmetric distribution of weight along the body of the swimmer. Second is the role of noise around the long axis which is manifested in rotational diffusion around the long axis, i.e., a type of wobbling [38-40] motion superimposed on the back and forth rotation about the long axis Figure 4D is a schematic of the phenomenon we propose, which could explain the anomalous behavior of swimmer P2. During the experiments, the swimmers (due to gravity) settle down at a distance of 1-2 microns away from the surface. Now, the idea relies on the fact that the swimmers while being rotated by the applied magnetic drive, can move along the vertical direction when their moments are not perfectly perpendicular to their long axis; thus, resulting in a periodic motion along the vertical direction. The sedimentation time (approximately at a rate of $9 \times 10^{-6} \mathrm{~m} / \mathrm{s}$ ) for a typical distance of 1 micron is about $0.11 \mathrm{~s}$. In one limit, when the magnetic field frequency is lower than $10 \mathrm{~Hz}$, the swimmer would be going up and down (vertically) while rotating about its own axis due to the reciprocal drive. At lower frequencies, as shown in Figure 4D, in one cycle of oscillation, half of the swimmer approaches the surface whereas the other half is fairly unaffected. This prevents the swimmer from being affected by surface uniformly across the body length. In the other limit, when the frequency is higher, the swimmer would remain in the same z-plane. Thus, at higher frequencies, swimmers are affected by the surface uniformly throughout the body length. So, during the event of turning, the balance of torques due to gravity and magnetic moment is also affected by presence of surface uniformly across the body. This is evident from Figure 3B where after $10 \mathrm{~Hz}$, the two propellers seem to follow different trends; with P2 showing variation in $q$.

\section{REFERENCES}

1. Lauga E, Powers TR. The hydrodynamics of swimming microorganisms. Rep Prog Phys (2009) 72:096601. doi:10.1088/0034-4885/72/9/096601

2. Purcell EM. Life at low Reynolds number. Am J Phys (1977) 45:3-11. doi:10. $1119 / 1.10903$

3. Lauga E. Life around the scallop theorem. Soft Matter (2011a) 7:3060-5. doi:10. 1039/C0SM00953A

4. Turner L, Ryu WS, Berg HC. Real-time imaging of fluorescent flagellar filaments. J Bacteriol (2000) 182:2793-801. doi:10.1128/jb.182.10.2793-2801. 2000

\section{CONCLUSION}

We present experimental and numerical study of magnetically driven helical swimmers at higher magnetic drive than what has been applied before. As explained in previous papers, under oscillating magnetic fields, these swimmers show back and forth motion due to interplay of magnetic torque and weight asymmetry. Their diffusivities can be significantly higher than the passively diffusing (not driven) structures due to the role played by orientational diffusion. The reciprocal sequence can be imperfect, determined by a parameter called degree of randomness, which results in even higher diffusivity. Here, we have shown a new method to determine the degree of randomness experimentally and have shown this parameter can be affected by the presence of the surface. This has resulted in anomalous increase in diffusivity in certain swimmers. It will be interesting to check how a collection of swimmers behave in dense suspensions where they interact with each other through hydrodynamic forces.

\section{DATA AVAILABILITY STATEMENT}

The raw data supporting the conclusions of this article will be made available by the authors, without undue reservation.

\section{AUTHOR CONTRIBUTIONS}

AG and GP developed the idea. GP performed the experiments and numerical simulations. Both the authors discussed extensively during this research and prepared the manuscript together. AG supervised the work.

\section{ACKNOWLEDGMENTS}

The authors would like to thank Pranay Mandal for helpful discussions and contributions in the building of experimental setup. We gratefully acknowledge the Micro and Nano Characterization Facility (MNCF, CeNSE) and National Nano Fabrication Center (NNFC, CeNSE) at IISc. We thank SERB for funding this research. We also acknowledge funding from MHRD, MeitY and DST Nano Mission for supporting the facilities at CeNSE.

5. Ghosh A, Fischer P. Controlled propulsion of artificial magnetic nanostructured propellers. Nano Lett (2009) 9:2243-5. doi:10.1021/nl900186w

6. Fischer P, Ghosh A. Magnetically actuated propulsion at low Reynolds numbers: towards nanoscale control. Nanoscale (2011) 3:557-63. doi:10.1039/c0nr00566e

7. Dreyfus R, Baudry J, Roper ML, Fermigier M, Stone HA, Bibette J. Microscopic artificial swimmers. Nature (2005) 437:862-5. doi:10.1038/nature04090

8. Jang B, Gutman E, Stucki N, Seitz BF, Wendel-García PD, Newton T, et al. Undulatory locomotion of magnetic multilink nanoswimmers. Nano Lett (2015) 15:4829-33. doi:10.1021/acs.nanolett.5b01981

9. Lauga E, Bartolo D. No many-scallop theorem: collective locomotion of reciprocal swimmers. Phys Rev E Stat Nonlinear Soft Matter Phys (2008) 78: 030901. doi:10.1103/PhysRevE.78.030901 
10. Qiu T, Lee TC, Mark AG, Morozov KI, Münster R, Mierka O, et al. Swimming by reciprocal motion at low Reynolds number. Nat Commun (2014) 5:5119-8. doi:10.1038/ncomms6119

11. Keim NC, Garcia M, Arratia PE. Fluid elasticity can enable propulsion at low Reynolds number. Phys Fluids (2012) 24:081703. doi:10.1063/1.4746792

12. Montenegro-Johnson TD, Smith DJ, Loghin D. Physics of rheologically enhanced propulsion: different strokes in generalized Stokes. Phys Fluids (2013) 25:081903. doi:10.1063/1.4818640

13. Fu HC, Wolgemuth CW, Powers TR. Swimming speeds of filaments in nonlinearly viscoelastic fluids. Phys Fluids (2009) 21:33102. doi:10.1063/1. 3086320

14. Pal M, Somalwar N, Singh A, Bhat R, Eswarappa SM, Saini DK, et al. Maneuverability of magnetic nanomotors inside living cells. Adv Mater (2018) 30:1800429. doi:10.1002/adma.201800429

15. Venugopalan PL, Sai R, Chandorkar Y, Basu B, Shivashankar S, Ghosh A. Conformal cytocompatible ferrite coatings facilitate the realization of a nanovoyager in human blood. Nano Lett (2014) 14:1968-75. doi:10.1021/ nl404815q

16. Ghosh S, Ghosh A. Mobile nanotweezers for active colloidal manipulation. Sci Robot (2018) 3:eaaq0076. doi:10.1126/scirobotics.aaq0076

17. Venugopalan PL, Jain S, Shivashankar S, Ghosh A. Single coating of zinc ferrite renders magnetic nanomotors therapeutic and stable against agglomeration. Nanoscale (2018) 10:2327-32. doi:10.1039/c7nr08291f

18. Mhanna R, Qiu F, Zhang L, Ding Y, Sugihara K, Zenobi-Wong M, et al. Artificial bacterial flagella for remote-controlled targeted single-cell drug delivery. Small (2014) 10:1953-7. doi:10.1002/smll.201303538

19. Dasgupta D, Pally D, Saini DK, Bhat R, Ghosh A. Nanomotors sense local physicochemical heterogeneities in tumor microenvironments. Angew Chem Int Ed Engl (2020) 59:23690-23696. doi:10.1002/anie.202008681

20. Ghosh A, Dasgupta D, Pal M, Morozov KI, Leshansky AM, Ghosh A. Helical nanomachines as mobile viscometers. Adv Funct Mater (2018) 28:1705687. doi:10.1002/adfm.201705687

21. Pal M, Dasgupta D, Somalwar N, Reshma V, Tiwari M, Teja D, et al. Helical nanobots as mechanical probes of intra- and extracellular environments. J Phys Condens Matter (2020) 32:224001. doi:10.1088/1361-648X/ab6f89

22. Keaveny EE, Walker SW, Shelley MJ. Optimization of chiral structures for microscale propulsion. Nano Lett (2013) 13:531-7. doi:10.1021/nl3040477

23. Paxton WF, Kistler KC, Olmeda CC, Sen A, St Angelo SK, Cao Y, et al. Catalytic nanomotors: autonomous movement of striped nanorods. J Am Chem Soc (2004) 126:13424-31. doi:10.1021/ja047697z

24. Bricard A, Caussin JB, Desreumaux N, Dauchot O, Bartolo D. Emergence of macroscopic directed motion in populations of motile colloids. Nature (2013) 503:95-8. doi:10.1038/nature12673

25. Mandal P, Patil G, Kakoty H, Ghosh A. Magnetic active matter based on helical propulsion. Acc Chem Res (2018) 51:2689-98. doi:10.1021/acs.accounts. $8 \mathrm{~b} 00315$

26. Mandal P, Ghosh A. Observation of enhanced diffusivity in magnetically powered reciprocal swimmers. Phys Rev Lett (2013) 111:248101. doi:10.1103/ PhysRevLett.111.248101
27. Mandal P, Chopra V, Ghosh A. Independent positioning of magnetic nanomotors. ACS Nano (2015) 9:4717-25. doi:10.1021/acsnano.5b01518

28. Lauga E. Enhanced diffusion by reciprocal swimming. Phys Rev Lett (2011b) 106:178101. doi:10.1103/PhysRevLett.106.178101

29. Vicsek T, Czirók A, Ben-Jacob E, Cohen I, Shochet O. Novel type of phase transition in a system of self-driven particles. Phys Rev Lett (1995) 75:1226. doi:10.1103/PhysRevLett.75.1226

30. Bechinger C, Di Leonardo R, Löwen H, Reichhardt C, Volpe G, Volpe G. Active particles in complex and crowded environments. Rev Mod Phys (2016) 88:045006. doi:10.1103/RevModPhys.88.045006

31. Wioland H, Woodhouse FG, Dunkel J, Kessler JO, Goldstein RE. Confinement stabilizes a bacterial suspension into a spiral vortex. Phys Rev Lett (2013) 110: 268102. doi:10.1103/PhysRevLett.110.268102

32. Volpe G, Buttinoni I, Vogt D, Kümmerer HJ, Bechinger C. Microswimmers in patterned environments. Soft Matter (2011) 7:8810-5. doi:10.1039/C1SM05960B

33. Kümmel F, ten Hagen B, Wittkowski R, Buttinoni I, Eichhorn R, Volpe G, et al. Circular motion of asymmetric self-propelling particles. Phys Rev Lett (2013) 110:198302. doi:10.1103/PhysRevLett.110.198302

34. Lauga E, DiLuzio WR, Whitesides GM, Stone HA. Swimming in circles: motion of bacteria near solid boundaries. Biophys J (2006) 90:400-12. doi:10. 1529/biophysj.105.069401

35. Bricard A, Caussin JB, Das D, Savoie C, Chikkadi V, Shitara K, et al. Emergent vortices in populations of colloidal rollers. Nat Commun (2015) 6:7470-8. doi: $10.1038 /$ ncomms 8470

36. Hawkeye MM, Brett MJ. Glancing angle deposition: fabrication, properties, and applications of micro-and nanostructured thin films. J Vac Sci Technol (2007) 25:1317-35. 10.1116/1.2764082

37. Johnson Singh H, Ghosh A. Porous three dimensional arrays of plasmonic nanoparticles. J Phys Chem C (2012) 116:19467-71. 10.1021/jp3062702

38. Ghosh A, Paria D, Singh HJ, Venugopalan PL, Ghosh A. Dynamical configurations and bistability of helical nanostructures under external torque. Phys Rev E - Stat Nonlinear Soft Matter Phys (2012) 86:031401. doi:10.1103/PhysRevE.86.031401

39. Ghosh A, Mandal P, Karmakar S, Ghosh A. Analytical theory and stability analysis of an elongated nanoscale object under external torque. Phys Chem Chem Phys (2013) 15:10817-23. doi:10.1039/c3cp50701g

40. Morozov KI, Leshansky AM. The chiral magnetic nanomotors. Nanoscale (2014) 6:1580-8. doi:10.1039/c3nr04853e

Conflict of Interest: The authors declare that the research was conducted in the absence of any commercial or financial relationships that could be construed as a potential conflict of interest.

Copyright (c) 2021 Patil and Ghosh. This is an open-access article distributed under the terms of the Creative Commons Attribution License (CC BY). The use, distribution or reproduction in other forums is permitted, provided the original author(s) and the copyright owner(s) are credited and that the original publication in this journal is cited, in accordance with accepted academic practice. No use distribution or reproduction is permitted which does not comply with these terms. 